\title{
Diet and coronary heart disease in England and Wales during and after the second world war
}

\author{
D J P BARKER AND C OSMOND
}

From the MRC Environmental Epidemiology Unit, University of Southampton, Southampton General Hospital, Southampton SO9 $4 X Y$

SUMMARY During the second world war there were large changes in consumption of fats, fibre, and sugar in Britain. These changes matched recent recommendations made by the Committee on Medical Aspects of Food Policy (COMA) with the object of reducing the incidence of coronary heart disease (CHD). It is widely believed that CHD mortality fell during the war. This paper re-examines CHD mortality among middle-aged people in England and Wales from 1931 to 1967. After allowance for changes in the rules for coding cause of death, and for the sharp increase in all-causes mortality in 1940, there is little to suggest that time trends in CHD were much influenced by the war. Because of confounding variables, this does not argue against the COMA report. However, it gives no support to the view that compliance with the recommendations on fat, fibre, and sugar will lead, by itself, to an appreciable fall in CHD mortality in middle-aged people.

The recent report from the Committee on Medical Aspects of Food Policy (COMA) recommends dietary changes in the United Kingdom with the object of reducing the incidence of coronary heart disease. ${ }^{1}$ The recommendations include reduction in total fat intake, no further increase in intake of simple sugars but an increase in consumption of fibre-rich carbohydrates such as bread, cereals, fruit, and vegetables.

During the second world war government food policy effected major and widespread changes in the British diet. Fat and sugar consumption fell sharply ${ }^{2}$ and fibre consumption rose. ${ }^{3}$ It is therefore relevant to examine changes in mortality from coronary heart disease (CHD) during and after the war. Previous analyses have concluded either that CHD mortality fell ${ }^{4}$ or that its pre-war increase was slowed. ${ }^{5}$ In 1949 the Registrar General wrote that whatever causes were responsible for the rapid increase in degenerative heart conditions during 1921-39 "must have been largely removed during the war". Such conclusions have been widely quoted.

\section{Method}

Interpretation of war-time rates in young adults is complicated by the large numbers of men who were in the armed forces overseas. In older age-groups diagnosis of cause of death is often less accurate. This paper therefore presents mortality rates for the civilian population aged 45-64 years.

In the Registrar General's annual reviews ${ }^{6}$ deaths in England and Wales were coded under the fourth revision of the International Classification of Disease (ICD) during 1931-39 and the fifth revision during $1940-49$. The change in classification in 1940 was accompanied by a change in coding procedure whereby, when more than one cause of death was mentioned on the certificate, rules of allocation of the underlying cause were abandoned in favour of the certifying doctors' stated preference. In order to allow comparison between the old and new classifications and coding procedures the Registrar General coded all 1939 deaths to the fourth and fifth revisions. In both classifications ICD 93 was "diseases of the myocardium" and ICD 94 was "diseases of the coronary arteries, angina pectoris". It was agreed that allocation to one or other of these codes was often arbitrary. ${ }^{57}$ The table shows the numbers of 1939 deaths, at ages $45-64$, coded to these causes under the rules of the fourth and fifth revisions. Under the fifth revision 2455 fewer deaths are coded to ICD 93 and 488 fewer to ICD 94 . Most of these deaths were coded as bronchitis (ICD 106) for there were 2855 certificates on which bronchitis was given as the cause of death with myocardial disease as a contributory or secondary cause. The table shows that under the fifth revision $\mathbf{4 0 7 6}$ more 
deaths were coded to bronchitis than under the fourth revision. These changes reflect the withdrawal of rules of allocation administered under the fourth revision, specifically "diseases of the heart (nos 90-95) or kidney (nos 130-133) to be preferred to any disease of the respiratory system (nos 104-114)".8

Number of deaths in 1939 in England and Wales coded to myocardial disease, coronary artery disease, and bronchitis under the fourth and fifth ICD revisions, men and women aged 45-64 years

\begin{tabular}{|c|c|c|c|c|c|}
\hline \multirow[b]{2}{*}{ Code } & \multirow[b]{2}{*}{ Description } & \multicolumn{2}{|l|}{ Men } & \multicolumn{2}{|l|}{ Women } \\
\hline & & $I C D 4$ & ICD 5 & $I C D 4$ & $I C D 5$ \\
\hline $\begin{array}{l}93 \\
94\end{array}$ & $\begin{array}{l}\text { Diseases of the myocardium } \\
\text { Diseases of the coronary arteries, }\end{array}$ & 8960 & 7147 & 6844 & 6202 \\
\hline & angina pectoris & 5498 & 5155 & 1705 & 1560 \\
\hline 106 & Bronchitis & 2432 & 5189 & 918 & 2237 \\
\hline
\end{tabular}

During 1950-57 deaths in England and Wales were coded to the sixth ICD revision. As in 1939, the Registrar General coded all 1949 deaths under both the existing classification and the new one. Under the fifth revision 22799 deaths were coded to ICD 93-94. Under the sixth revision 22854 deaths were coded to ICD 420-422, "arteriosclerotic and degenerative heart disease". The trends in ICD 93-94 from 1949 can therefore be extended into trends in ICD $420-422$ from 1950 to 1957. The trends from 1957 can be extended into the period 1958-67 when deaths were coded to the seventh ICD revision but with minimal changes in classification or coding procedure for CHD. ${ }^{9}$

\section{Results}

Figure 1 shows data as published by the Registrar General, that is, deaths coded to ICD 93 and 94 under the fourth revision during 1931-39 and under the fifth revision during 1940-49. In both men and women, mortality from diseases of the myocardium (ICD 93) rose to a peak in the late 1930s and thereafter declined, the steepest decline occurring in the war years. In contrast, mortality for coronary artery disease (ICD 94) rose progressively throughout the period except for a small decline in the early war years. As would be expected, rates were lower among women than men.

Figure 2 shows combined rates for myocardial and coronary artery disease during 1931-49 with rates for arteriosclerotic and degenerative heart disease (ICD 420-422) during 1950-67. Interrupted lines represent the 1931-39 rates as published by the
Registrar General, that is, coded to the fourth ICD revision. The dual coding of the 1939 data allows calculation of the proportion of these deaths, in each five-year age-sex group, which would have been coded to other causes (mostly bronchitis) under the multiple cause coding rules which applied under the fifth revision from 1940 onwards. These proportions have been applied to the age-sex specific rates to give estimates for each year from 1931 to 1939 . Continuous lines therefore represent the data for the entire 1931-49 period adjusted to the fifth ICD revision. A similar method, based on the dual coding of the 1949 and 1957 data, was used to adjust the 1950-67 data to the fifth ICD revision. An interrupted line shows the data as published, which are closely similar.

For men, the 1931-67 data as published showed a steep rise to a peak in 1939 , followed by an abrupt reversal and fall. After 1942 there was again a rise, but the rates did not reach those in 1939 until the 1950s. Adjusted to the fifth revision, however, the rates rose until 1940, fell in 1941 and 1942, and thereafter rose almost without interruption unti1963. Among women, the data as published showect a slow rise to a peak in 1936-40, a fall in 1941-42 and little change thereafter. The adjusted rates peak in 1940.

As would be expected from the data on bronchitio shown in the table, adjustment of the 1931-39 deathe increases the pre-war mortality rates for alf respiratory diseases combined (ICD 104-114)? Figure 3 shows that even after adjustment there was a striking increase in respiratory deaths among men in 1940. The rise was smaller in women, among whom rates were lower throughout.

There was a similarly abrupt rise in total mortality and infant mortality in 1940 (figs 4 and 5). In both sexes death rates from all causes other than myocardial and coronary artery disease, and violence (ICD 163-198), rose sharply in 1940 (fig 4). Despite the overall decline in mortality during $1931-49$, the 1940 rate for men was the highest during the period. This 1940 peak resulted from an increase in death rates from a range of diseases, including respiratory diseases and infections. Similarly, infant mortality (fig 5) rose sharply in 1940 and again in 1941 before resuming its downward trend. There were four peaks in deaths attributed to influenza (ICD 11, fourth revision, ICD 33 fifth revision) during 1931-49, of which one was in 1940 (fig 6).

\section{Discussion}

Although deaths attributed to CHD rose steeply and almost without interruption during 1931-49(fig 1), it was generally agreed that diagnostic transfer 


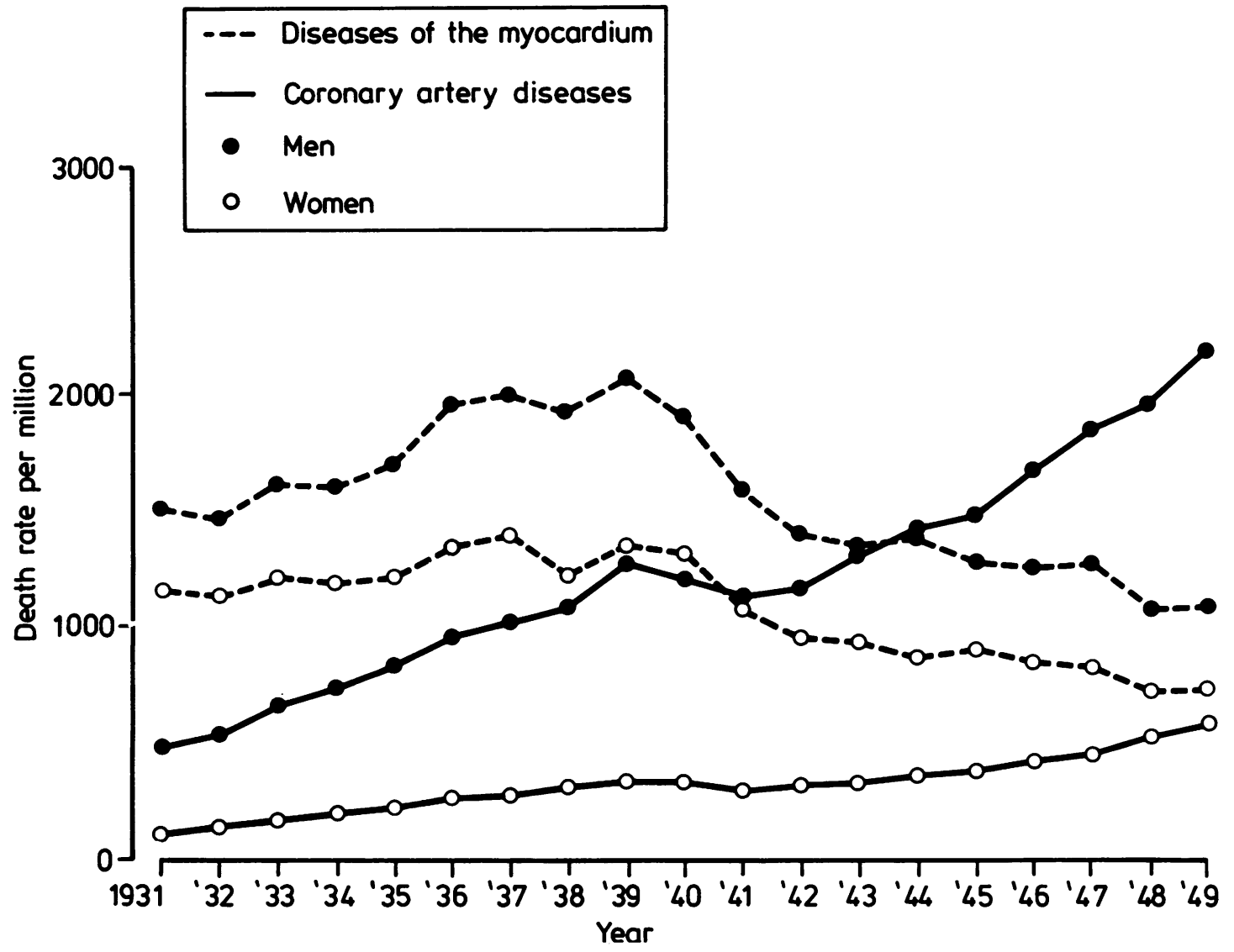

Fig 1 Annual mortality from myocardial disease and coronary artery disease (ICD 93 and 94 , fourth and fifth revisions) in England and Wales, men and women aged 45-64 years, 1931-49.

between this classification and diseases of the myocardium had occurred. Hence in the analyses of CHD trends by Ryle and Russell, ${ }^{7}$ Morris, ${ }^{5}$ and others, the two causes of death were considered together. The results, based on data coded under the fourth and fifth ICD revisions, led to the conclusions that during the war CHD mortality fell ${ }^{4}$ or that its pre-war increase was slowed. ${ }^{5}$ These changes have been attributed to a beneficial effect of the war-time diet. Our re-examination of CHD mortality in middle-aged men and women brings into question both the changes that occurred and their relation to diet.

In a review of mortality trends in England and Wales, Campbell noted that revision of the ICD in 1940 , and a change in the rules of allocation for deaths with multiple causes, complicated interpretation of the trends at that time. ${ }^{10}$ Our adjustment of the 1931-39 data to the fifth ICD revision is based on certain assumptions, including that of a fixed proportion of heart disease deaths accompanied by respiratory disease throughout the period. Nevertheless the adjusted data shown in fig 2 (continuous lines) seem likely to be more accurate indicators of trends than the data originally published. Adjusting male deaths changes the apparent peak in 1939 to a peak in 1940 and reduces the magnitude of the subsequent decline in relation to pre-war levels: the 1942 rate, the lowest during and after the war, is similar to those in 1937-38. In women, there is similarly a 1940 peak in the adjusted data.

We have shown that 1940 was a year of unusually high mortality from a range of diseases, not only among adults (figs 3 and 4) but among infants as well-for whom 1941 was also an unusual year (fig 5). 


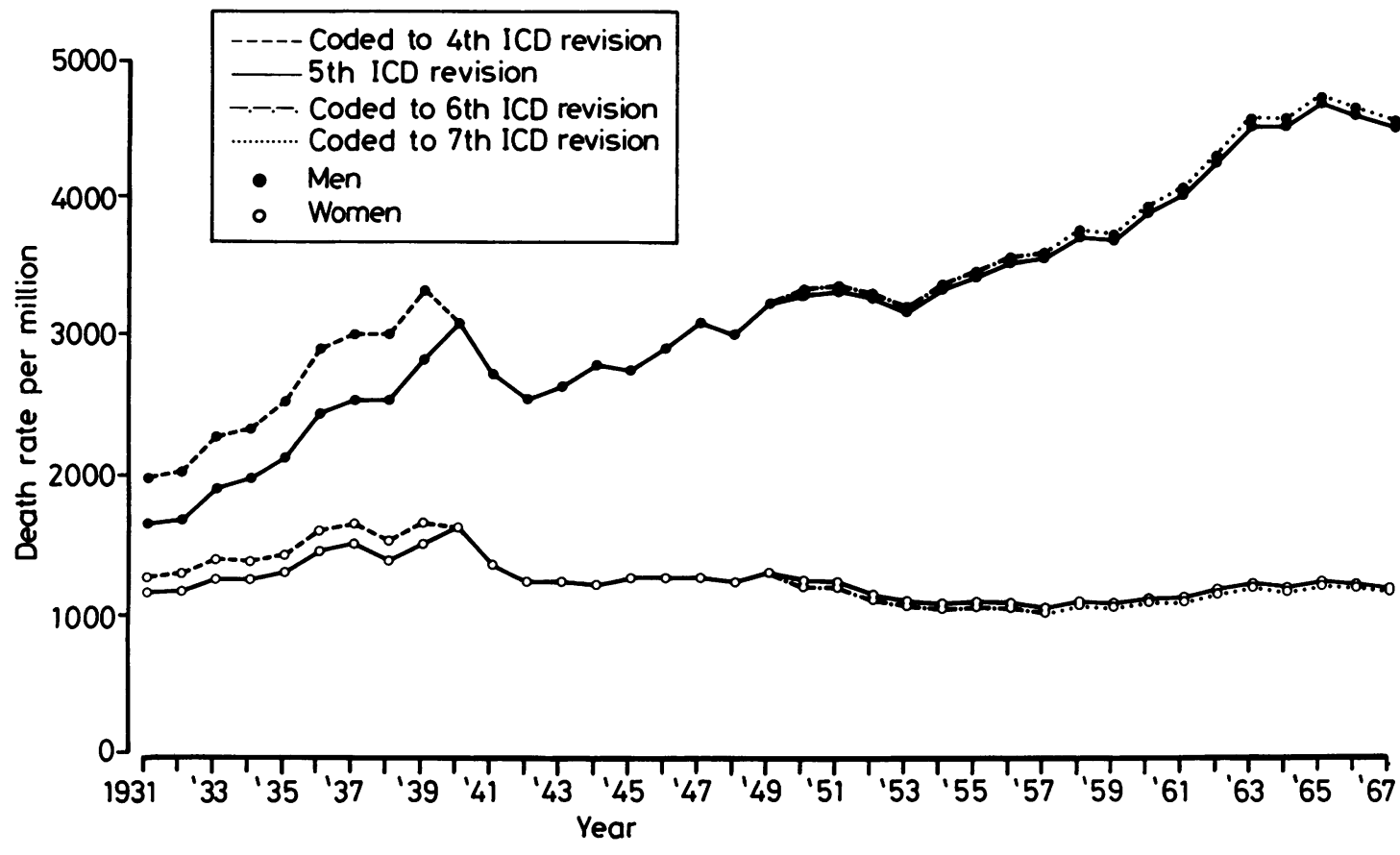

Fig 2 Annual mortality from myocardial disease and coronary artery disease (ICD 93 and 94) 1931-49 and arterioscleroticand degenerative heart disease (ICD 420-422) 1950-67 in England and Wales, men and women aged 45-64 years with rates adjusted to fifth ICD revision (see text).

Contributing to this was an influenza epidemic (fig 6) and a cold winter. ${ }^{40}$ Given this unusual mortality, and the sharp worsening in environmental conditions consequent upon the outbreak of war, it seems reasonable to exclude 1940 when considering trends in CHD.

Taking the continuous lines in fig 2 as the best estimates of CHD mortality, and putting aside the 1940 rates, there is no evidence that time trends in CHD were much influenced by the war. Among men there was a modest fall from 1939 to 1942 such that the 1942 rate was similar to that in 1938 . Thereafter the rise in mortality continued steadily until the mid-1960s. Among women there was similarly a fall from 1939 to 1942 . Thereafter there was little change.

Wartime and immediate post-war trends in younger and older age groups are similar to those in the middle-aged, but their interpretation is less secure because of large scale recruitment of young men into the armed forces and less accuracy of death certification in the elderly.

The wartime dietary changes were large and, because of rationing, widespread. The findings of the
Combined Food Board, which compared estimates of consumption during 1943-44 with those during 1934-38, were summarised as follows. "Important increases were: for potatoes $45 \%$, vegetables $34 \%$, milk and milk products $28 \%$ and grain products $17 \%$. Important decreases were in tomatoes and citrus fruits $50 \%$, other fruits $44 \%$, poultry and fish $39 \%$, sugar $31 \%$, meat $21 \%$ and fats $16 \%$." These large changes did not take place immediately on the outbreak of war. The decline in food importation and increase in home production occurred over one to two years. Not until April 1942 was the extraction rate of flour raised from $68-75 \%$ to $85 \%$, with the consequent sharp increase in cereal fibre consumption. Food controls persisted after the war. From 1949 to 1950 they were gradually withdrawn, and rationing finally ended in 1954 .

Levels of consumption of fat, fibre, and sugar during the 1940s differed markedly from those in the 1930 s and 1950s. Total fat consumption fell from $39 \%$ of food energy in $1934-38$ to $36 \%$ in 1941 and $33 \%$ in 1947 . It returned to $38 \%$ in 1950 and increased thereafter. ${ }^{2}$ Wartime levels may be compared with the reduction to $35 \%$ recommended 


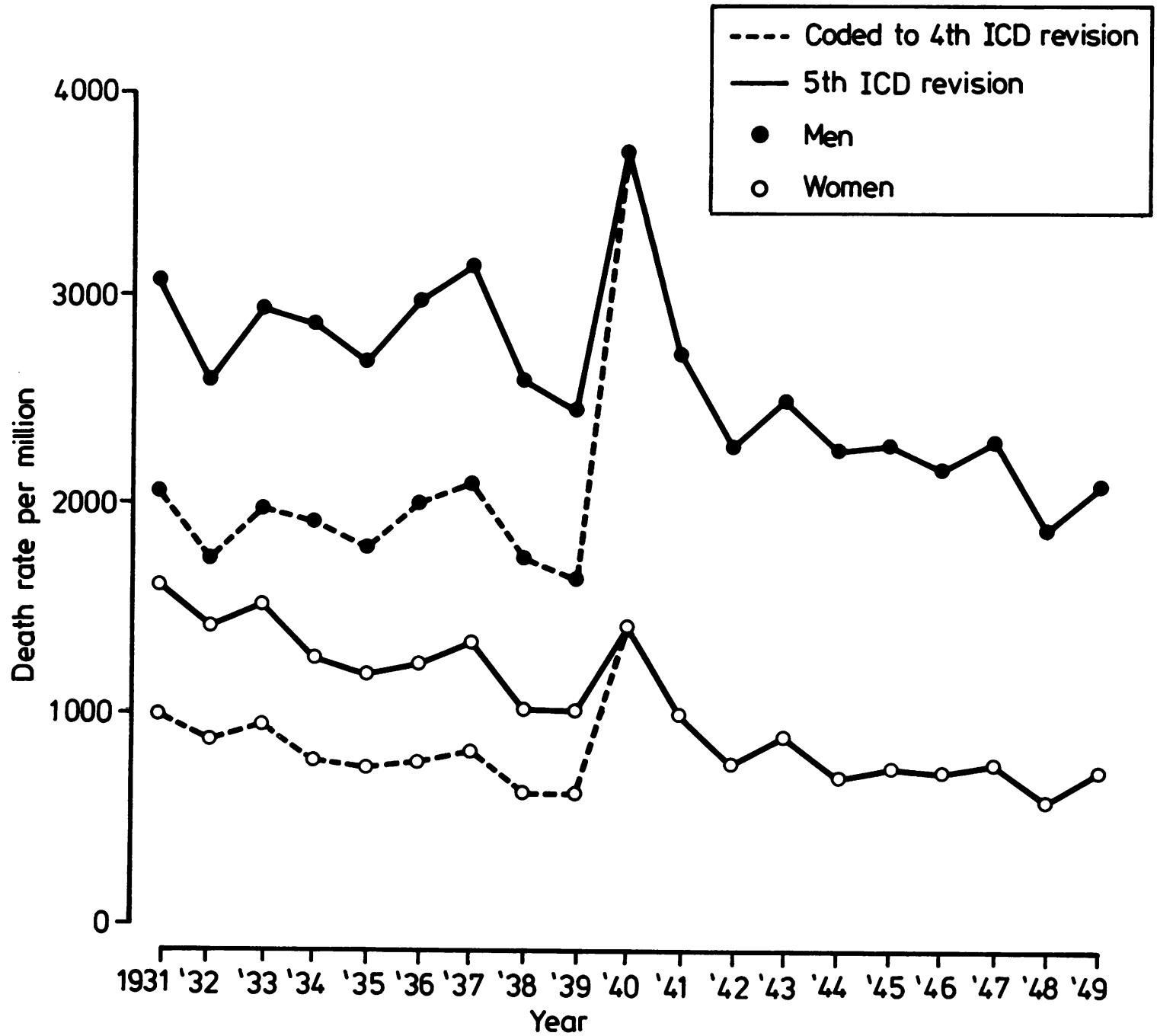

Fig 3 Annual mortality from respiratory disease (ICD 104-114) in England and Wales, men and women aged $45-64$ years with 1931-39 rates adjusted to fifth ICD revision (see text).

by COMA. Using current food tables, the estimated ratio of polyunsaturated to saturated fatty acids was 0.26 and therefore did not reach the COMA recommended level of $0 \cdot 45 .{ }^{12}$ Estimated cereal fibre consumption rose from $9 \mathrm{~g}$ per person per day in 1938 to $19-25 \mathrm{~g}$ in $1944 .^{3}$ During the same period vegetable fibre consumption rose from $10 \cdot 1 \mathrm{~g}$ per person per day to $13 \cdot 2 \mathrm{~g}$. Consumption of both cereal and vegetable fibre fell back after the war, the former to pre-war levels. Sugar consumption fell from $44 \mathrm{~kg}$ per person per year in 1938 to $30 \mathrm{~kg}$ in 1941 . It returned to pre-war levels around 1953 and increased thereafter. ${ }^{2}$

These changes in consumption of fat, fibre, and sugar cannot be readily reconciled with the trends in CHD mortality (fig 2). It could be argued that the decline in mortality from 1939 to 1942 was a response to the changes. The mechanism for so immediate a response would presumably be a change in blood coagulability rather than reduced deposition of atheroma. However, the changes were introduced gradually, over the first one to two years of the war, 


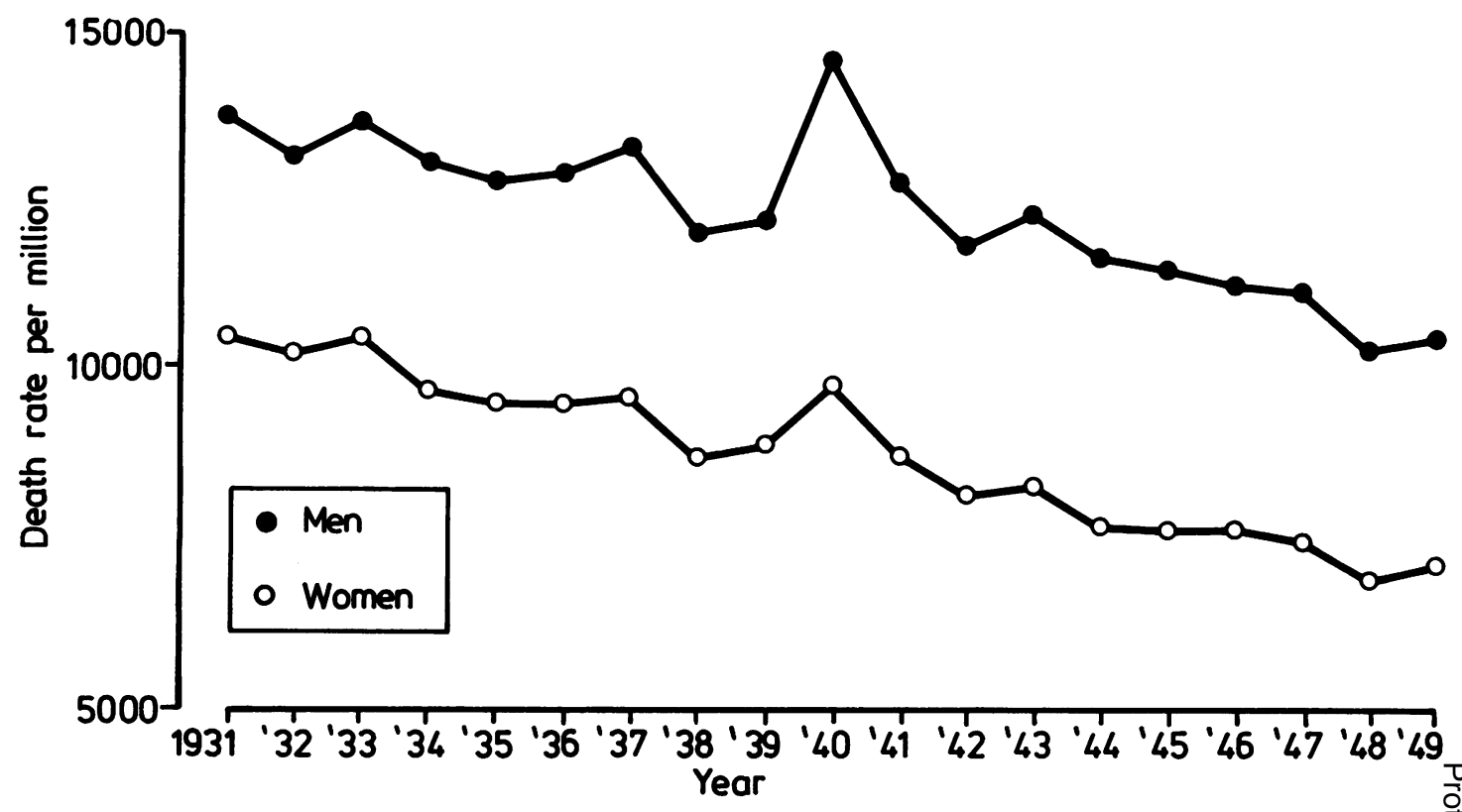

Fig 4 Annual mortality from all causes, other than myocardial and coronary artery disease and violence, in England and Wales, men and women aged 45-64 years, 1931-49.

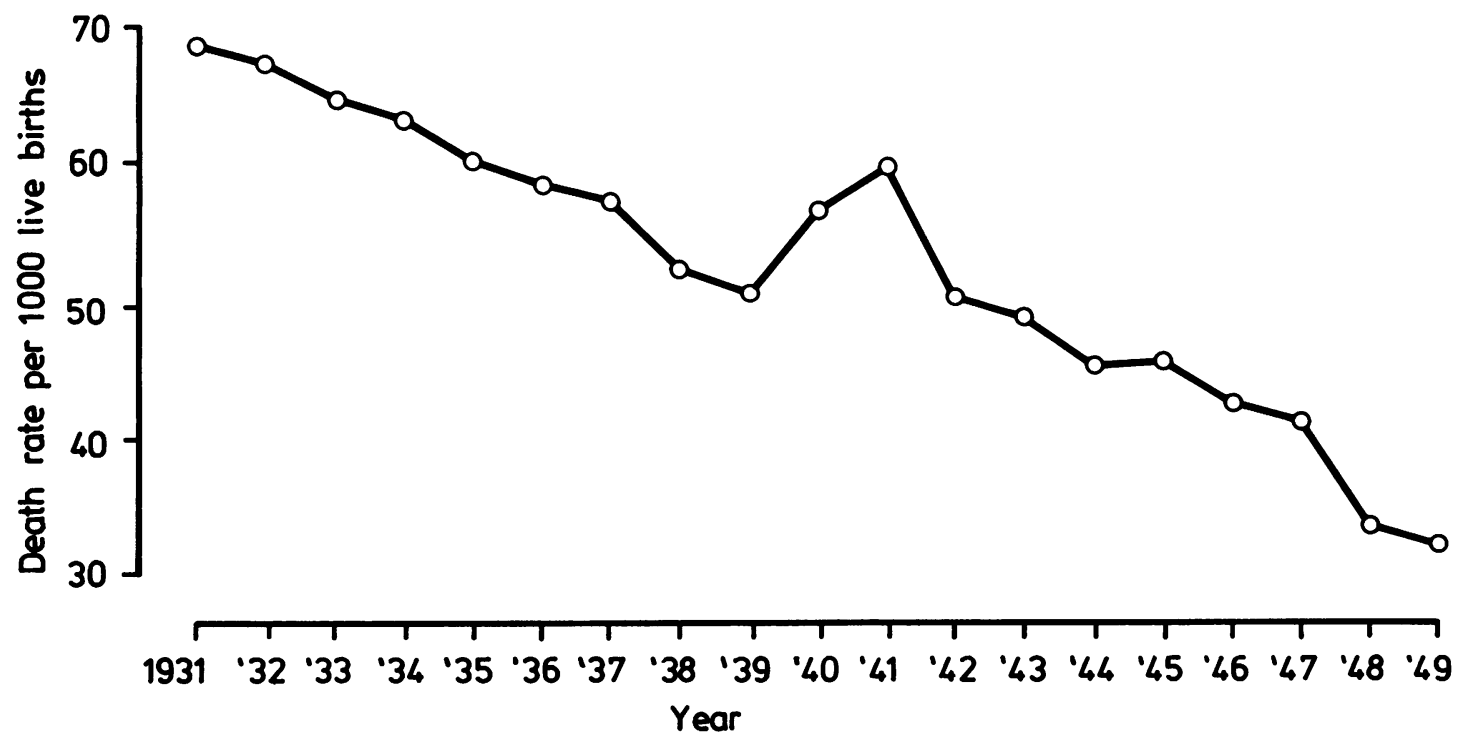

Fig 5 Annual infant mortality in England and Wales, 1931-49. 


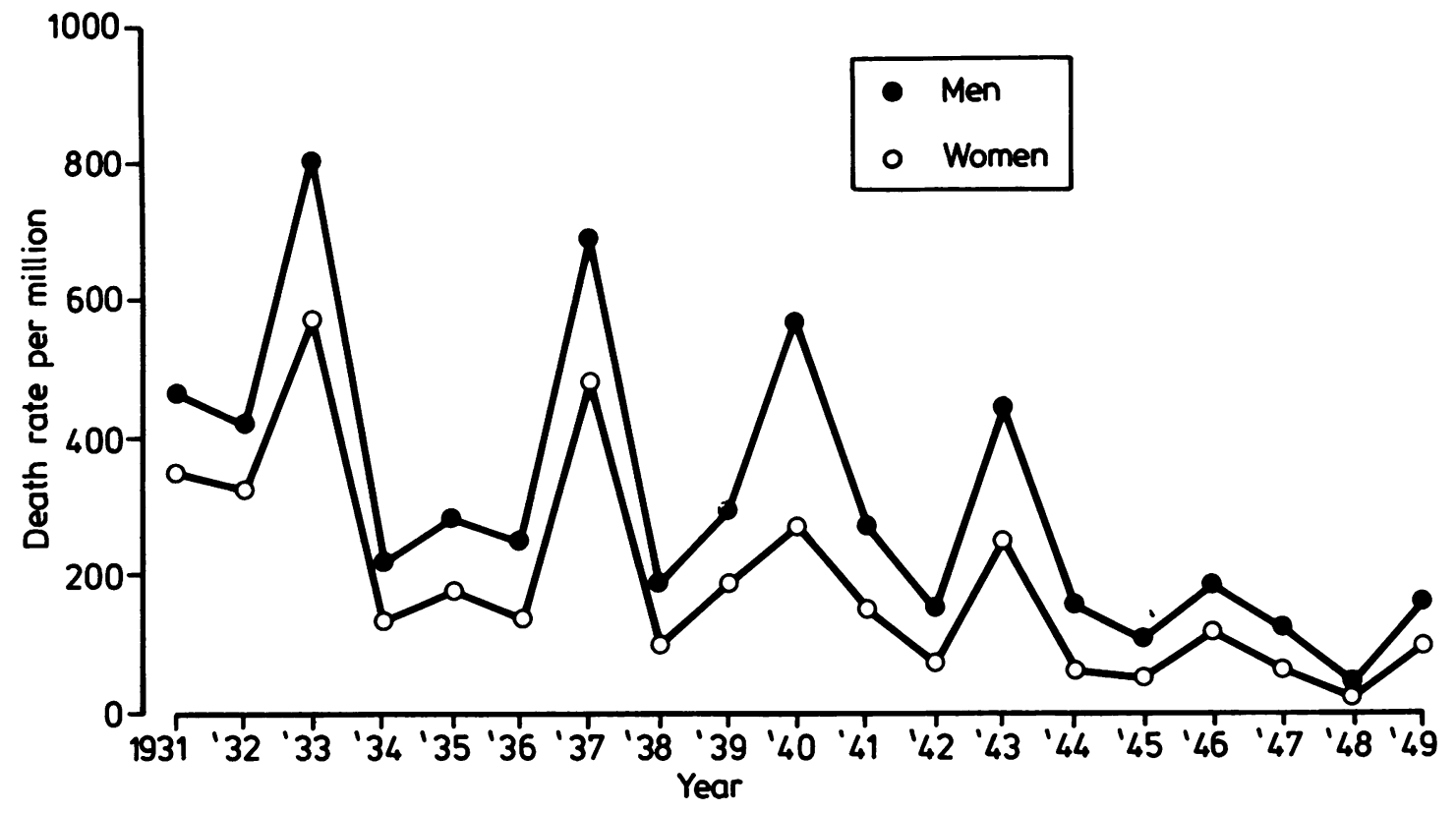

Fig 6 Annual mortality from influenza (ICD 11, fourth revision, 33 fift revision) in England and Wales, men and women aged 45-64 years, $1931-49$.

and food controls persisted throughout the 1940 s. There is therefore no evident explanation for the resumed rise after 1942 nor for the lack of an acceleration of the rise after the withdrawal of food controls in the 1950s. If it is argued that the wartime dietary changes prevented what would otherwise have been a steeper rise, the lack of response to removal of food controls is similarly unexplained. The proposition of a latent period of some years before dietary change is followed by a reduction in mortality does not lessen the incongruity between the dietary and CHD mortality trends. If, however, the lateni period was of the order of 10 years or more, benefits to middle-aged people would be reflected in mortality among the elderly. Figure 2 would reflect the wartime diets of young men, many of whom were in the armed forces.

Comparison of wartime trends in CHD mortality and changes in estimated per capita consumption of fat, fibre, and sugar cannot give conclusive evidence. Certified cause of death is an imperfect indicator of mortality caused by CHD. Exposure to other causative influences, dietary and non-dietary, may have changed during the war. Stress, for example, may have increased, but so too may muscular exercise. ${ }^{11}$ Cigarette consumption rose to a peak in 1946, fell during 1947-49 and thereafter continued to rise. ${ }^{13}$ We conclude that, while our findings do not argue against the COMA report, they do not support the view that compliance with the recommendations on fat, fibre, and sugar consumption will lead, by itself, to an appreciable fall in CHD mortality in middle-aged men.

We are grateful to Miss Clare Pippard who helped with abstraction of the data and to Mrs Bridget Wilde who typed the manuscript.

\section{References}

${ }^{1}$ Department of Health and Social Security. Report on Health and Social Subjects 28. Diet and cardiovascular disease. London: HMSO, 1984.

${ }^{2}$ Greaves JP, Hollingsworth DF. Trends in food consumption in the United Kingdom. World Rev Nutr Diet 1966; 6: 34-89.

${ }^{3}$ Southgate DAT, Bingham S, Robertson J. Dietary fibre in the British diet. Nature 1978; 274: 51-2.

'The Registrar General's Statistical Review of England and Wales for the six years 1940-45: Vol 1, 1940-45. London: HMSO, 1949. 
${ }^{5}$ Morris JN. Recent history of coronary disease Lancet 1951; 1: 69-73.

${ }^{6}$ The Registrar General's Statistical Review of England and Wales: Part 1 Tables, Medical, 1931-49. London: HMSO.

${ }^{7}$ Ryle JA, Russell WT. The natural history of coronary disease. Br Heart J 1949; 11: 370-89.

${ }^{8}$ Manual of the International List of Causes of Death: based on the fifth decennial revision by the International Commission, Paris, 1938. London: HMSO, 1940.

${ }^{9}$ Clayton DG, Taylor D, Shaper AG. Trends in heart disease in England and Wales, 1950-73. Health Trends 1977; 9: 1-6.
${ }^{10} \mathrm{Campbell} \mathrm{H}$. Changes in mortality trends in England and Wales, 1931-1961. Washington DC: US Department of Health, Education and Welfare, 1965.

${ }^{11}$ Magee HE. Application of nutrition to public health. $\mathrm{Br}$ Med J 1946; 1: 475-82.

${ }^{12}$ Studies in urban household diets 1944-49, second report of the National Food Survey Commitee. London: HMSO, 1956.

${ }^{13}$ Lee PN, ed. Statistics of smoking in the United Kingdom. London: Tobacco Research Council, 1976. 